\title{
A REVIEW OF EPIDEMIOLOGICAL STUDIES OF ORAL CANCER AND PRECANCER IN MALAYSIA
}

R.B. Zain, N. Ghazali. A review of epidemiological studies of oral cancer and precancer in Malaysia. Annal Dent Univ Malaya 2001; 8: 50-56.

\section{ABSTRACT}

This paper attempts to review epidemiological studies of oral cancer and precancer in Malaysia. The definitions of prevalence, incidence, risk habits and oral cancer and precancers were discussed to better understand the different types of studies conducted, which would be important in making comparisons between studies.

Currently, epidemiological data on oral cancer in Malaysia are sketchy. The only incidence data for oral cancer in Malaysia was reported by Hirayama in 1966, 35 years ago. He estimated that 3.1 new cases per 100,000 population were diagnosed for the year 1963 .

A number of histopathological data of oral and maxillofacial biopsies were reported. Oral cancer accounted for one-fifth of all oral biopsies. A national study on oral mucosal lesions in Malaysia carried out in 1993/4 reported that there was a variation seen in the occurrence of oral premalignancy among the ethnic groups. The Indians and the indigenous people of Sabah and Sarawak were identified as high risk groups for oral cancer and precancer. It was also observed that both of the ethnic groups chewed betel quid.

In conclusion, the epidemiological studies have provided useful data, which may be used in planning for future oral health programmes and research towards enhancing Malaysia's on-going effort in preventing the occurrence of these diseases.

Key words: Oral cancer, oral precancer, epidemiological studies.

\section{INTRODUCTION}

'Oral cancer epidemiology' is a study of the distribution of oral cancer in human populations, which is undertaken primarily for two purposes. Firstly, epidemiological studies aim to search for the determinants of oral cancer. Well designed epidemiological studies have established that the risk of oral cancer varies with time, place and individual population characteristics. This has led the researchers to search for more defined environmental exposures acting in the pathogenesis of oral cancer or the differential susceptibility of a population to such exposures. Secondly, epidemiological data provides a strong basis for the rational planning of healthcare activities. This information validates the prioritisation of healthcare services within a community. With tailoring of healthcare provision, it is possible to monitor
Review Article

\author{
R.B. Zain ${ }^{1}$ and N. Ghazali ${ }^{2}$ \\ ${ }^{1}$ Department of Oral Pathology, \\ Oral Medicine and Periodontology \\ Faculty of Dentistry, University of Malaya \\ Kuala Lumpur, Malaysia \\ ${ }^{2}$ Department of Oral \& Maxillofacial Surgery, \\ Faculty of Dentistry, \\ National University of Malaysia \\ Kuala Lumpur, Malaysia
}

Corresponding author - Rosnah Binti Zain

the results and impact of secondary preventive interventions on the scale and trend of the disease in a population over time.

From a global perspective, epidemiological studies have indicated that oral cancer (140-145, ICD-9) is fast becoming an important form of cancer. In many parts of Asia, oral cancer continues to be a major health problem, where it constitutes one of the most common forms of cancer reported annually (1). The Ministry of Health Malaysia in 1996, reported that lip and oral cancer were the third most common cancer deaths in government hospitals (2). Therefore it can be regarded as an important health problem in Malaysia

Over the years, numerous epidemiological studies of oral cancer have been conducted in Malaysia. These studies have contributed significantly to the current understanding of the disease and successfully highlighted specific risk factors affecting different sections of the population.

With the turn of a new century, it would be useful to trace and reflect upon the passage and development of oral cancer epidemiological research in Malaysia so as to provide a coherent basis for future researchers in this field. This paper summarises aspects of descriptive and analytical epidemiology derived from published literature on oral cancer and precancer in Malaysia. In addition, this review also touches upon pertinent methodological issues that have significant bearing in the planning, performing and outcome of epidemiological studies.

\section{GENERAL CONSIDERATIONS}

\section{Malaysia at a Glance}

Malaysia is situated in Southeast Asia and abuts the neighboring countries of Thailand, Indonesia and Singapore. The total expanse of Malaysia covers a land area of 329,758 square kilometres. Malaysia is often referred to as being made up of two main geographical 
regions namely, Peninsular Malaysia and East Malaysia, which is composed of the states of Sabah and Sarawak situated in the north of Borneo. Based on the preliminary count report of the 'Population and Housing Census of Malaysia 2000', the population of Malaysia is 22.2 million, 11.2 million men $(50.5 \%)$ and 11.0 million women $(49.5 \%)$ in year 2000 (3).

Malaysia is a multiethnic society. Based on the midyear population estimates of the 1991 census, the distribution of the ethnic groups in Malaysia are: the Malays, $47.0 \%$; the indigenous people of Sabah and Sarawak (among the major groups in Sarawak are Bidayu, Iban, Melanau and among the major groups in Sabah are the Kadazan, Bajau and Murut), $11.1 \%$; the Chinese, $24.3 \%$; the Indians, $6.9 \%$; the other ethnic groups, $3.2 \%$ and the non-Malaysians, $7.5 \%$ (4).

\section{Re-visiting Definition and Criteria in Epidemiological Studies of Oral Cancer and Precancer}

\section{a. Definitions of epidemiological terminology}

At this stage, there is a need to highlight the confusion observed in some publications on epidemiology of oral cancer owing to the indiscriminate use of terms in describing oral cancer frequency. Prevalence and incidence are measures of cancer frequency. However, it must be noted that each term connotes a specific epidemiological implication and a publication resulting from the haphazard use of terminology by a researcher is in danger of misleading the reader.

Prevalence of cancer quantifies the proportion of individuals in a population who have cancer at a specific instant. It provides an estimate of the probability that an individual will have cancer at a point in time and is therefore useful for identifying risk groups within the population studied. In contrast, incidence of cancer quantifies the number of new cancer cases that develop in a defined population during a specified time interval. Hence, the incidence of cancer indicates the size of the cancer problem experienced within that population. Subsequent recording of incidence over specified time intervals i.e. trends provides a means of studying the impact of various environmental factors on the development of cancer and the monitoring of the intervention of primary and secondary preventive activities (5).

Many epidemiological studies have used the terms incidence and prevalence to mean what is more accurately described as 'relative frequency'. Relative frequency for oral cancer can be defined as the frequency of oral cancer as compared to other cancers or other diseases.

\section{b. Measuring incidence and prevalence}

The ideal method for determining the incidence of cancer in a defined population over a specified time interval is through the establishment of a populationbased cancer registry. It is ironic that Western countries with a relatively lower frequency of oral cancer have established population-based cancer registries while many developing countries with higher frequencies of oral cancer do not. The reasons behind this are attributed to various circumstances such as political instability, lack of funding and lack of expertise.

\section{c. Definition of oral cancer and precancer}

Many epidemiological studies have failed to look at the importance of precisely defining 'oral cancer'. The International Classification of Diseases (ICD) was originally developed by the World Health Organisation (WHO) for the purpose of statistical analysis of various diseases. The ICD has now become the basis for global standardisation in the definition of oral cancer. The ICD is a coding system based on the anatomical origin of the primary cancer. In this system, specific areas related to the mouth are given with specific ICD codes, which include the lips, tongue, floor of mouth, soft palate, buccal mucosa, and gingiva/alveolus (including the retromolar region)(6). Despite the ICD codes, oral cancer is still known to have remarkable diversity in its definition. Unlike other anatomical regions of the body, the boundaries of the oral cavity are not always clearly demarcated, making an exact definition of oral cancer by site almost an impossible task to achieve (7).

In the absence of an international consensus on the definition of oral cancer, epidemiological studies have resorted to use of various definitions, which are based on a combination of selected ICD sites. For example, oral cancer is a combination of : 141 , ICD-9 (tongue) and 143-145, ICD-9 (gum, floor of mouth and other unspecified parts of the mouth). By affiliating selected ICD sites under a single group, a larger sample size may be obtained for a group. This approach also alleviates the difficulty in the determination of the primary site of oral lesion, particularly in large lesions that project into several ICD-recognised anatomical sites $(8,9)$. The main setback to this approach is the possibility of one site exerting a 'masking effect' over another site within a group resulting in a distortion of the true epidemiological picture. This is particularly significant when one site is excessively more common compared with its other affiliated sites.

Various revisions of the original ICD code have taken place several times and this may have contributed to the lack of consensus in reporting oral cancer. Currently, the ninth edition is frequently used in the majority of oral cancer papers although the most current revision is the tenth edition. The tenth edition contains some changes involving sites of oral cancer because these sites have now been recognised to have distinctive aetiological and epidemiological patterns (10). Due to the widespread use of the ninth edition as a basis of oral cancer, it may be wise to prepare two sets of data for evaluation. One in the ninth edition so that meaningful comparisons can be made with other studies and another version based on the tenth edition for future use when the ninth edition becomes obsolete. Despite the uncertainty emanating from the lack of consensus, the fundamental take-home message is that a precise definition based on the ICD must accompany any terminology chosen for use in the study. 
Oral precancer presents its own set of rules in relation to definition. Oral precancer consists of oral precancerous lesions and oral precancerous conditions. The definition of oral precancerous lesion is 'a morphologically altered tissue in which cancer is more likely to occur than in its apparently normal counterpart' (11). Oral precancerous lesions have been further subcategorised by the World Health Organisation (1997) into the clinical and histological classification. This subcategorisation seemed necessary to avoid the misconception that certain oral precancerous lesions can be histologically confirmed and is considered by some to be synonymous with squamous epithelial dysplasia. The clinically recognised oral precancerous lesions are leukoplakia, erythroplakia and 'palatal keratosis associated with reverse smoking' (WHO, 1997). The term 'potentially malignant lesions or conditions' (12) has more currently been preferred as the clinically recognised lesions above, in the majority of cases, do not become malignant. Any of the above clinical diagnoses will be further accepted as oral precancerous lesions histologically, if they are squamous epithelial dysplasia, squamous cell carcinoma-in-situ and solar keratosis (11). These definitions and criteria are especially important in gaining valid epidemiological data, which can be compared globally between one study and another.

The oral precancerous condition has been defined as 'a generalised state associated with a significantly increased risk of cancer' (11). The oral precancerous conditions recognised are sideropenic dysphagia, lichen planus, oral submucous fibrosis, syphilis, discoid lupus erythematosus, xeroderma pigmentosum and epidermolysis bullosa (WHO, 1997).

\section{d. Standardising terminologies on risk habits}

Oral cancer is a disease resulting from the long-term culmination of mucosal exposure to various carcinogens and co-factors. Established risk factors of oral cancer include tobacco usage and areca nut chewing habit, while alcohol consumption has been recognised as an important co-factor.

Many studies in the Asia Pacific region reported that some cultural habits cause changes to the oral mucosa. The mucosal changes observed in this region have been attributed mainly to tobacco smoking and/or quid chewing habit (13). The term 'quid' in this paper means "a substance or mixture of substances placed in the mouth for long topical contact with the mucosa, and containing two basic ingredients, tobacco and/or areca nut, in raw or any manufactured or processed form"(14). Some of the tobacco smoking changes presents as benign/reactive lesions such as smoker's palate, leukoedema and smoker's melanosis. Many mucosal changes arising from quid chewing are cancerous and precancerous lesions such as oral carcinoma, leukoplakia and erythroplakia or conditions such as oral lichen planus and oral submucous fibrosis.

There are also other lesions seen in quid chewers where its precancerous status is still questionable such as chewers' mucosa, betel quid lichenoid lesions and quid-induced lesions which include tobacco-lime user's lesions, snuff induced lesions and areca quid lesions (14). These lesions, until now, have been considered as benign lesions due to the lack of evidence to support them as premalignant lesions.

Studies on oral cancer and precancer are many, with variations from one study to another (15). The cancerous and precancerous lesions/conditions as described above, have been observed in Malaysia and have been similarly attributed to quid chewing by many researchers. The purpose of this paper is to review the epidemiological studies of oral cancer and precancer in Malaysia.

\section{EPIDEMIOLOGY OF ORAL CANCER AND PRECANCER IN MALAYSIA}

\section{Prevalence and Incidence}

To date, there is only one report, by Hirayama (1966), on the incidence of oral cancer in Malaysia (16). Hirayama estimated that the incidence of oral cancer was 3.1 new cases per 100,000 population for the year 1963 . He found that the incidence was highest among the Indian ethnic group. Hirayama also observed that the incidence of oral cancer varied between individual states of Peninsular Malaysia, where the highest rate was observed in Selangor (8.2 per 100,000) while the states of Kelantan, Terengganu and Kedah had the lowest rates of 1.9 or less. This was probably due to a higher proportion of the Indian ethnic group residing in Selangor compared with the states of Kelantan, Terengganu and Kedah. Two hospital-based studies reported in 1973 using convenience samples, that is, an outpatient specialist clinic and medical personnel in hospitals, have also identified the relatively higher occurrence of oral precancers and cancers in the Indian ethnic group $(17,18)$.

The ethnic variation of oral cancer was also observed by Ramanathan et al in 1976 (19) and $\mathrm{Ng}$ et al in 1985 (20) based on a series of biopsies sent to the Institute of Medical Research (IMR). Their studies showed that oral cancers were higher among the Indian as compared to other ethnic groups in Malaysia. However, these studies were case series of biopsies, hence they could not be generalised to the population. Nevertheless, they represent frequencies of oral cancer as compared to other pathologies received by the IMR. Despite the fact that the IMR receives biopsies from almost the whole of Malaysia, an oral cancer incidence for Malaysia in the later years is still not possible. This is partly due to the decentralisation of oral cancer cases to other general pathology laboratories throughout the country thus deterring accurate evaluation of the true occurrence rate of oral cancer. Although the IMR reports could not be generalised, its result could be used towards rationalising the need for population-based studies to further determine the risk groups.

The prevalence of oral cancer in Peninsular Malaysia was first reported in 1976 . Only $0.01 \%$ of the 9076 subjects examined in 1973/74 had oral cancer (21). This 1976 population survey on oral lesions in Peninsular 
Malaysia further supports this ethnic variation and the Indian ethnic was identified as a high risk group, where the prevalence for the study population of both oral cancer and precancer was $1.33 \%$ (21).

In 1993/94, another population study on oral mucosal lesions was carried out in Malaysia. Out of 11,697 subjects examined, the prevalence of oral cancer was found to be $0.04 \%$ (22). This report also formally authenticated the variation seen in the occurrence of oral premalignancy among the ethnic groups. In addition, this study further identified another section of the Malaysian population to be at risk of developing oral cancer. The indigenous people of Sabah and Sarawak were also identified as a group which had a high occurrence of precancerous (15.4\%) and cancerous lesions (1.9\%) (22).

Since 1963, there are no incidence studies on oral cancer and precancer in Malaysia (16). The prevalence of oral cancer and precancer reported this far showed a wide variation where the prevalence of oral precancer such as leukoplakia varies from 1.3 to $26 \%$ (15). These variations may be due to differences in sample selection where studies include samples with predominance of certain ethnic, sex and age groups. Other causes of variations were the criteria used and examiner variability. Many studies on oral mucosal lesions uses the WHO criteria as well as many examiners. However, most of these studies do not describe proper training and calibration procedures in using and interpreting these criteria. The level of accuracy in detecting/not detecting the lesions by examiners was also not available.

The prevalence of oral cancer appears to increase from $0.01 \%$ in $1973 / 74$ to $0.04 \%$ in $1993 / 94(21,22)$. In view that both these studies used similar sampling procedures and criteria for lesions, this may indicate that there is a true increase in oral cancer prevalence. However, the increase can be attributed to the fact that the later study includes the states of Sabah and Sarawak and thus these studies are not comparable. The 1993/ 94 survey also reported that oral cancer was observed among the indigenous people of Sabah and Sarawak (out of the five subjects found to have oral cancer, two of them were the indigenous people of Sabah and Sarawak) (23).

Oral precancer prevalence (in particular leukoplakia) was seen to decrease from 1973/74(1.3\%) to $1993 / 94$ $(0.96 \%)$. For both studies, the criteria used for leukoplakia was based on the WHO criteria. However, the decrease may not be a true decrease as, despite using similar criteria, the method of training and calibrating examiners may differ leading to variation on the interpretation of criteria. For example, without properly training the examiners to differentiate between the white lesions which are precancerous (e.g. leukoplakia) from the white lesions which are not precancerous (e.g. frictional keratosis) will lead to under or over diagnosis. The fact that the 1993/94 study includes Sabah and Sarawak also makes these prevalence incomparable with that of $1974 / 75(21,22)$.

\section{Risk habits for oral cancer and precancer}

It had been recognised worldwide that tobacco smoking, quid chewing and alcohol consumption are the three main risk habits found to be associated with oral cancer and precancer $(1,8)$. The following section of this paper will briefly discuss these habits in Malaysia.

\section{Smoking habits in Malaysia}

Tobacco smoking in Malaysia is in many forms. Smoking the commercial brand cigarettes seemed to be the most common form. Other manufactured types of tobacco smoking are the 'bidi' used mainly by the Indians, handmade paper-rolled cigarette (raw tobacco rolled in special paper prior to smoking) or leaf-rolled cigarettes called 'rokok daun'(raw tobacco rolled in temburna leaves prior to smoking) used by some rural Malays and an Indonesian imported type of cigarette (kretek) containing spices such as cloves in addition to the tobacco (24).

\section{Prevalence of tobacco smoking in Malaysia}

The dental epidemiological adult survey of Peninsular Malaysia (1976) reported the prevalence of current smokers among those aged 15 and above in Peninsular Malaysia was found to be $23.2 \%$, whereas the First National Health and Morbidity Survey (NHMS \#1) on adults of the same age cohorts and location reported $21.5 \%$ of current smokers (25). In 1993/94, the nationwide oral mucosal lesions survey showed a prevalence of $23.2 \%$ of current smokers aged 25 years and above (22) and in 1996, the current smokers aged 18 and above, were $24.8 \%$ (Second National Health and Morbidity Survey - NHMS \#2 (25). The latter 2 surveys were conducted for the whole of Malaysia (Peninsular Malaysia, Sarawak and Sabah). The NHMS \#2 also showed that the highest prevalence of current smokers were among the Malays and the indigenous people of Sabah and Sarawak; males; lower level of education and low household income.

\section{Quid chewing habits in Malaysia}

The quid chewing habit appears to be a dying habit among the younger generation and urbanites in Malaysia. However, it is still widely practiced by some sections of the population including the Indians working in plantations remote from an urban centre, the indigenous people of Sabah and Sarawak and some elderly Malays living in rural villages (26-28). The main ingredients used are areca nut (seed of Areca catechu), betel-leaf (leaf of Piper betle) and lime (boiled from seashells) (19). Tobacco is added to the quid mixtures especially among the Indians and the indigenous people of Sabah and Sarawak. The Malay quid chewers do not include tobacco in their quid (28). The indigenous people of Sabah and Sarawak and some of the Indians (24) add gambir (a preparation from leaves and twigs of the shrub Uncaria gambir) (19) to the quid.

\section{Prevalence of quid chewing in Malaysia}

The prevalence of betel-quid chewers was 3.9\% for Peninsular Malaysia in 1974/75 (21) and $6.97 \%$ for 
Peninsular Malaysia, Sabah and Sarawak in 1993/94 (22). The Indians, the Malays and the indigenous people of Sabah and Sarawak are found to indulge in betel-quid chewing habit (22). Most Chinese do not indulge in quid chewing habit. Chinese who have indulged in this habit are probably those who have integrated into the Indian families.

\section{Alcohol consumption in Malaysia}

Many ethnic groups in Malaysia consume beer and stout. Special home-brands such as toddy and samsu are used by the Indians and domestically manufactured rice alcohol are used by the indigenous people of Sarawak (24)

\section{Prevalence of alcohol intake in Malaysia}

Data on the prevalence of alcohol drinking habit in Malaysians is limited. This could be due to the ethnic and religious sensitivity with regards to drinking alcoholic beverages. The prevalence of alcohol drinking habit was $4.2 \%$ (23). The highest prevalence of alcohol drinking habit was found to be among the Indians (13\%), followed by the indigenous people of Sabah and Sarawak (10\%) and the Chinese (7.8\%) (22). A recent survey in 1996 conducted among non-Muslims showed a 23\% prevalence of current drinkers (2). The Malays are Muslims and drinking alcoholic beverage is prohibited. The 1993/94 data further showed that the alcoholic drinking habits are higher in men $(8.5 \%)$ than in women. This trend is especially so for the Indians and the Chinese (22). It is however, interesting to note that there was a much higher prevalence of alcohol drinking habit among women as compared to men among the indigenous people of Sarawak (29).

Risk habits and prevalence of oral cancer in Malaysia

The relationship of betel quid chewing and oral cancer in Malaysia has been recognised since 1960s (30). In 1963, Hirayama (1966), compared the prevalence of oral pharyngeal cancers in Southeast Asia and Central Asia concluded that the ethnic differences observed in the prevalence of oral cancer were partially explained by the quid chewing habit (16). Hirayama further conducted a hospital based, case-control study in Kuala Lumpur on the effect of chewing habits and found that there were more chewers in the cancer group compared to the control group and this result was similar in other Asian countries. The relationship between oral cancer and betel-quid chewing were further substantiated in retrospective studies of case series from two time periods at the Institute of Medical Research (IMR) namely the 1967 - 1972 period on 898 cases (16) and the $1978-1984$ period on 749 cases (17). Both studies showed that for those who chewed betel quid the main site of cancer was the buccal mucosa whereas for those who do not chew betel quid, the most prevalent sites were not the buccal mucosa.

$\mathrm{Ng}$ et al in 1986 (31) further reported that in a series of 100 cases of squamous cell carcinoma, $83 \%$ of all oral squamous cell carcinoma only had the betel quid chewing habit. The Indians were the majority with oral squamous cell carcinoma and practising betel quid chewing.

Most of the studies on risk habits and oral cancers in Malaysia, so far, were mainly large case series, which had shown strong indication that one of the risk factors was tobacco chewing in the form of betel quid. The high prevalence of oral cancer among the Indians compared to the other ethnic groups seemed to commensurate with the high prevalence of quid chewers among them (23). Despite having a high prevalence of quid chewers and smokers among the indigenous people of Sabah and Sarawak, data relating these factors with oral cancer are limited.

Studies correlating alcohol drinking and oral cancer in Malaysia are lacking. Ng et al (1986) in their series of 100 oral cancer cases reported that out of 41 oral cancer patients practising a single habit, only $5 \%$ had only the alcohol drinking habit. The majority of the oral cancer patients had multiple habits, which included alcohol-drinking (31).

\section{Risk habits and prevalence of oral precancer in Malaysia}

Many prevalence studies had been conducted on oral precancer (OPC) and risk habits such as betel quid chewing. The prevalence of OPC varies from $0.01 \%$ to $23.6 \%$ (15). Two cross-sectional studies conducted in 1973 by Ramanathan et al on 407 medical attendants/ assistants (17) and 1648 outpatients from three dental clinics (18) showed a correlation of anatomical sites (the buccal mucosa is the most frequent site) of these oral precancer with the quid chewing habits, which was similar to the oral cancer. Most of the studies on oral precancer were on selected Malaysian populations. Oral precancers were also commonly found among the Indians $(17,18,21,22,32)$ and the indigenous people of Sabah and Sarawak (22). The recent cross-sectional studies showed that the indulgence in tobacco related quid chewing practices for both groups, the Indians and the indigenous people of Sabah and Sarawak, were significantly related to oral precancers $(26,27,29)$. In comparison, in the Malay quid chewers (where tobacco was not part of their quid content), an equally low prevalence of oral precancer among the chewers and non-chewers was observed (28).

Similar to oral cancer, studies on the relationship between alcohol drinking habit and oral precancer in Malaysians are also lacking. Some population-based studies in Malaysia had reported on the prevalence of alcohol drinking habit but had not reported any relationship between alcohol drinking and oral precancer $(2,21,22)$

\section{CONCLUSION}

Studies in Malaysia, this far, had identified the high risk groups for oral precancer and the strong causal relationship with quid chewing. The multiethnic society of Malaysia with varying socio-cultural lifestyles can be the venue for further in-depth study on the possible 
chemopreventive agents and/or molecular markers for the prevention or early detection of oral precancers respectively. Any possible findings can be used to enhance Malaysia's on-going effort in trying to prevent the occurrence of these diseases.

\section{ACKNOWLEDGEMENT}

The authors would like to acknowledge Dr Khairiyah from the Dental Division, Ministry of Health Malaysia for her assistance towards the publication of this paper.

\section{REFERENCES}

1. Johnson NW. Orofacial neoplasms. Global epidemiology, risk factors and recommendations for research. Int Dent J 1991; 41: 365 - 375.

2. Annual Report 1996, Ministry of Health Malaysia. Kuala Lumpur. Ministry of Health Malaysia 1996; pp. $84-86$.

3. Department of Statistics Malaysia. Population and housing census of Malaysia 2000 - preliminary count report. Kuala Lumpur: Department of Statistics Malaysia 2000; pp. iii.

4. Department of Statistics Malaysia. Statistics Handbook of Malaysia 2000. Kuala Lumpur: Percetakan Malaysia Nasional Berhad 2000; pp. 3 $-6$.

5. Hennekens $\mathrm{CH}$, Buring JE. Epidemiology in Medicine. Boston/ Toronto. Little, Brown and Company 1987; pp. $64-65$.

6. World Health Organisation (ICD-9). The International Classification of Diseases, Ninth Revision. Geneva: World Health Organisation 1978.

7. Moore SR, Johnson NW, Pierce AM, Wilson DF. The epidemiology of mouth cancer: a review of global incidence. Oral Dis 2000; 6: 65-74.

8. Chen J, Katz RV, Krutchkof DJ. Intraoral squamous cell carcinoma. Epidemiologic patterns in Connecticut from 1935 to 1985 . Cancer 1990; 66: 1288-1296.
11. Pindborg JJ, Reichart PA, Smith CJ, van der Waal I and in Collaboration and pathologists in 9 countries. Histological Typing of Cancer and Precancer of the Oral Mucosa - World Health Organisation International. Histological Classification of Tumours. Berlin. Springer-Verlag 1997; pp. $21-31$.

12. Ogden GR, Macluskey M. An overview of the prevention of oral cancer and diagnostic markers of malignant change. 1. Prevention Dent Update 2000; 27: 95-99.

13. Zain RB. Cultural and dietary risk factors of oral cancer and precancer - a brief overview. Oral Oncol 200; 37: 205-210.

14. Zain RB, Ikeda N, Gupta PC, Warnakulasuriya S, van Wyk C, Shrestha P, Axéll T. Oral Mucosal lesions associated with betel-quid, areca nut and tobacco chewing habits: consensus from a workshop held in Kuala Lumpur, Malaysia, November 25 - 27, 1996. J Oral Pathol Med 1999; 28: 1-4.

15. Zain RB, Ghazali N, Raja Latifah RL. Methodologic Issues In Studies on Oral Precancers - Abstracts from the Second National Public Health Conference "Smart Partnerships In Public Health" (Abstract \# FPD1B5) 2001; pp. 122.

16. Hirayama T. An epidemiological Study of Oral and Pharyngeal Cancer in Central and SouthEast Asia. Bull Wld Hlth Org 1966; 34: 41 - 69.

17. Ramanathan K, Canagayagam A, Tan CK, Ratnanesan A. Frequency of oral precancerous conditions in 407 Malaysians with correlation to oral habits. Med J Malaysia 1973; 27: 173 - 181.

18. Ramanathan K, Tan CK, Ratnanesan A, Canagayagam A. Oral precancerous condition frequency in 1648 Malaysians with correlation to oral habits. Dent J Malaysia 1973; 13: 11 - 21.

19. Ramanathan K, Lakshimi S. Oral carcinoma in Peninsular Malaysia: Racial Variations in the Indians, Malays, Chinese, and Caucasians G.A.N.N. Monograph on Cancer Res 1976; 18: 27 36.

20. Ng KH. Siar $\mathrm{CH}$. Ramanathan K Murugasu P 
22. Zain RB, Ikeda N, Razak IA, Axëll T, Majid ZA, Gupta P, Yaacob M. A national epidemiological survey of oral mucosal lesions in Malaysia. Community Dent Oral Epidemiol 1997; 25: 377 383.

23. Zain RB, Ikeda N, Yaacob $M$. Oral mucosal lesions in Malaysia - an epidemiologic study. Petaling Jaya: Ministry of Health Malaysia, University of Malaya and Aichi Gakuin University 1995; pp. 37 - 38.

24. Zain RB. Nutritional and habitual factors for oral cancer and precancer among selected Malaysians (Indians, Malays and the Indigenous people of Sarawak). Kuala Lumpur: Faculty of Dentistry, University of Malaya 1999; pp. 5-100.

25. Haniza MA, Maimunah AH, Rusilawati J, Latipah S, Suraya A. National Health and Morbidity Survey 1996: Smoking among adults. Kuala Lumpur: Public Health Institute, Ministry of Health Malaysia 1999; (15): 23 .

26. Zain RB, Ikeda N, Shanmuhasuntharam P, Fukano H, Razak IA, Ghazali MN. Screening for oral cancer and precancer: A comparative study between urban and rural Indian estate populations in Malaysia. Abstract Handbook of the Joint Scientific meeting of the Royal College of Pathologists of Australasia 1997; pp. 83.
27. Rahman ZAA, Zain RB, Abang Z, Ikeda N, Razak IA, Fukano H, Abdullah M, Hii M. Oral mucosal lesions in Indigenous people of Sarawak and their related habits. J Dent Res (abstract no: 10) 1999; 78: 1171 .

28. Raman RA, Rahman ZAA, Zain RB, Samsuddin AR, Razak IA, Abdullah F, Venugopal I. Oral mucosal lesions in Malay quid and non-quid chewers in Kelantan. J Dent Res (abstract no: 9) 1999; 78: 1171.

29. Rahman ZAA, Zain RB, Zubidah A, Ikeda N, Razak IA, Aung S, Abdullah F. A Pilot Study of Oral Mucosal Lesions among Sarawak Indigenous People. Dent J Malaysia, 1997; 18: 26 - 29.

30. Marsden ATH. Betel Cancer in Malaya. Med J Malaysia 1960; 14: 162 - 165.

31. Ng KH, Siar CH, Ramanathan K, Murugasu P. A study of oral habits in 100 cases of squamous cell carcinoma in Malaysia. Ann Dent 1986; 45: 7 - 11.

32. Hashim BY. Betel-nut chewing and oral mucosal lesions. A preliminary study. Hosp Dent (Tokyo) 1991; 3: 13 - 15 . 